\title{
MODELING OF THE “PLAN DA MATTUN” ARCHAEOLOGICAL SITE USING A COMBINATION OF DIFFERENT SENSORS
}

\author{
D. Novák, P. Tokarczyk*, P.W. Theiler \\ Institute of Geodesy and Photogrammetry, ETH Zürich, 8093 Zürich, Switzerland \\ (david.novak, piotr.tokarczyk, pascal.theiler)@geod.baug.ethz.ch
}

\author{
Youth Forum
}

KEY WORDS: aerial, close range, DEM/DTM, laser scanning, measurement, reconstruction, visualization

\begin{abstract}
:
Plan da Mattun is located at 2200 metre above sea level in the Tasna valley in alpine south-eastern Switzerland. In this remote location, finds dating back to the time of Ötzi (3000 B.C.) were discovered by archaeologists from the University of Zurich. For detailed investigations of the site as well as for documentation and visualization purposes the archaeologists were interested in digital models of the terrain and of certain boulders. In the presented project a digital terrain model of the rock stream located at the beginning of the valley was created, as well as detailed models of four larger boulders. These boulders average to 15 metre in height and width. The roughness of terrain makes it difficult to access certain areas and requires using multiple surveying techniques in order to cover all objects of interest. Therefore the digital terrain model was acquired using a combination of terrestrial laser scanning (TLS) and photogrammetric recording from an unmanned aerial vehicle (UAV). The larger boulders were reconstructed with a combination of TLS, terrestrial and UAV-based photogrammetry. With this approach it was possible to acquire a highaccuracy dataset over an area of $0.12 \mathrm{~km}^{2}$ under difficult conditions. The dataset includes a digital terrain model with a ground sampling distance of $10 \mathrm{~cm}$ and a relative accuracy of $2 \mathrm{~cm}$ in moderately sloped terrain. The larger boulders feature a resolution of $1 \mathrm{~cm}$ and a relative accuracy of $0.5 \mathrm{~cm}$. The 3D data is to be used both for archaeological visualization purposes and for geological analysis of the rock stream.
\end{abstract}

\section{INTRODUCTION}

The Tasna valley is located in the eastern part of Switzerland in the Canton Graubünden amidst the Alps (Figure 1). At Plan da Mattun - the beginning of the valley - a large rock stream is located (Figure 2, left). The boulders (Figure 2, right) range from 2 up to 15 metre in height and width.

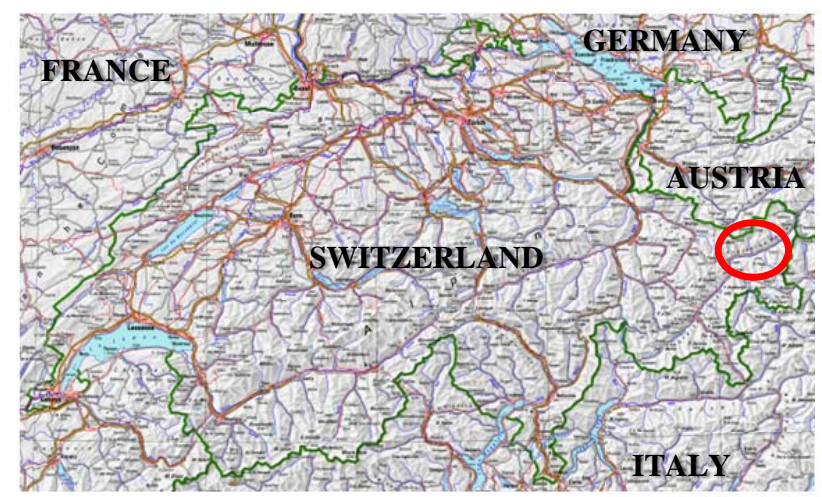

Figure 1: Location of the Archaeological Site, Plan da Mattun
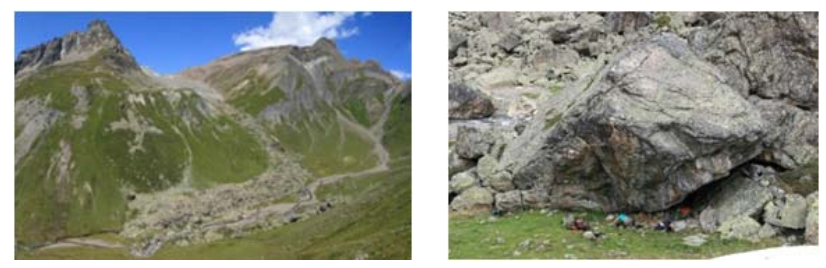

Figure 2: Objects of Interest

Left: Plan da Mattun, Rock Stream, Right: Boulder Example
The site is of great interest for the archaeologists of University of Zurich, due to the fact that many finds where discovered that date back to the time of Ötzi (3000 B.C.). These finds derive from early alpine dwellers, taking shelter below the overhanging boulders at Plan da Mattun while they were hunting or before crossing the mountain pass at the end of the valley.

The goal of our work was to obtain a digital surface model of the rock stream as well as high resolution models of four larger boulders that were of particular interest for the archaeologists. The resulting models can be used to document and analyse the finds and the surrounding location. The digital surface model was acquired using a combination of terrestrial laser scanning (TLS) and unmanned aerial vehicle (UAV) photogrammetry. The larger boulders were reconstructed with TLS as well as terrestrial and UAV-based photogrammetry.

To combine all the measurements into a common coordinate system, ground control points were measured with differential GNSS using a static reference station. The used coordinate system is the Swiss national reference frame (LV95). The control points were used during the generation of the digital surface model. To reference the detailed model of the larger boulders, the density of the reference network was increased using a total station and differential GNSS.

\section{DIGITAL SURFACE MODEL}

The goal of this step was to obtain a high resolution digital surface model (DSM) of the valley floor $\left(0.12 \mathrm{~km}^{2}\right)$. The model should also contain larger rock boulders. The conventional 
digital elevation models available at the Swiss federal office of topography (swisstopo) do not model the valley in sufficient detail - especially in areas with large boulders - which made it necessary to generate a new DSM with a ground sampling distance of $10 \mathrm{~cm}$.

\subsection{Terrestrial Laser Scanning}

The data acquisition for the generation of a DSM based on TLS was done using the Leica ScanStation C10. According to the manufacturer, measurements up to 300 metre are possible (albedo of 90\%). In this project, due to the bad intersection angle between ground based scanner positions and the ground itself, the reflected energy is mostly lower. An albedo of $20 \%$ results in a maximal range of 135 metre, which proved to be a realistic assumption. Range measurements between 1 metre and 50 metre have a standard deviation of $4 \mathrm{~mm}$. Considering also the standard deviation of angular measurements, the resulting point accuracy is around $6 \mathrm{~mm}$. Moreover, the scanner has an integrated 4 Megapixel digital camera, which is placed in the same optical axis as the emitted scanner beam and can be used to provide the colour information.

Because of the massive boulders on the whole site and the lack of an accurate surface model, the scan positions could not be planned in advance until the practical field inspection. Merely a coarse estimation of the number of required scans can be carried out considering the maximum range measurement of the scanner. Thus, the selection of suitable scanner positions was done in the field. Finally, as seen in Figure 3, 30 scans were taken.

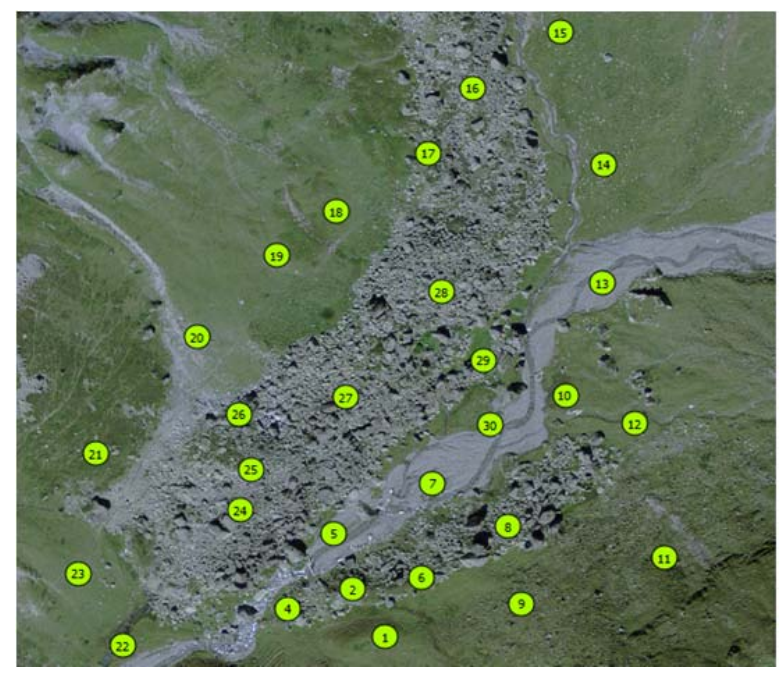

Figure 3: 30 Scan Positions for the Generation of the DSM

The relative and absolute pose estimation of the scans was accomplished simultaneously. The scanner positions were measured with differential GNSS. The ground control points or additionally temporary marked points - also measured with GNSS - were used to provide the orientation. Therefore, all scans are directly transformed into the reference frame.

The first processing steps were carried out in Cyclone software (Leica Geosystems). Each scan was coloured using the digital images from the integrated camera. The software provides a tool to accomplish this automatically. Because of the previously carried out orientation, the scans can be unified into one single point cloud without further processing. A cut-out of the coloured point cloud can be seen in Figure 4.

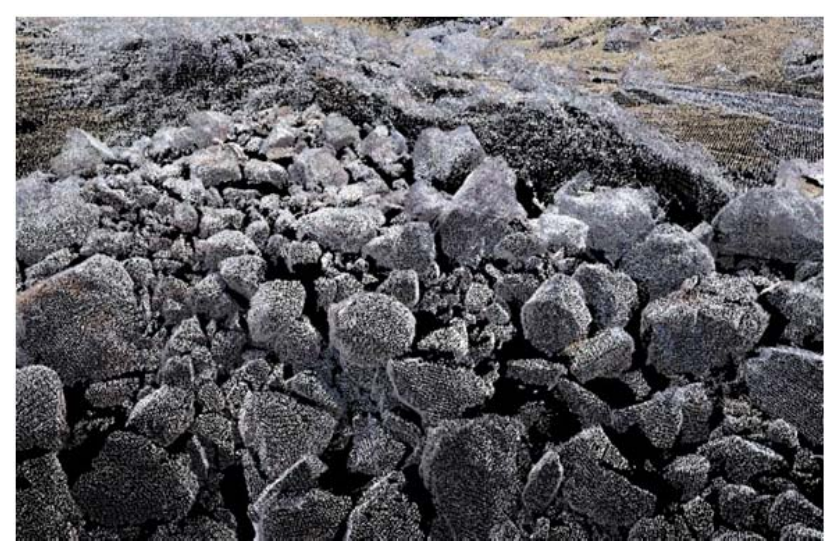

Figure 4: Coloured Point Cloud in Cyclone

The resulting point cloud consists of 130 million points, but there are regions with an exaggerated point density. Thus a reduction of the point cloud was carried out. With a mean point spacing of $10 \mathrm{~cm}$, the point cloud was reduced to 15 million points. Out of these points a raster image was generated using ArcGIS software (esri). The pixel size was again set to $10 \mathrm{~cm}$. During this step, the colour information is lost due to software limitations. Because of the bad quality of the scanner images, this fact was ignored. Instead, the orthoimage generated from the UAV images (see Chapter 2.2) was used for visualisation.

The resulting digital surface model overlaid with the orthoimage from the UAV images is shown in Figure 5.

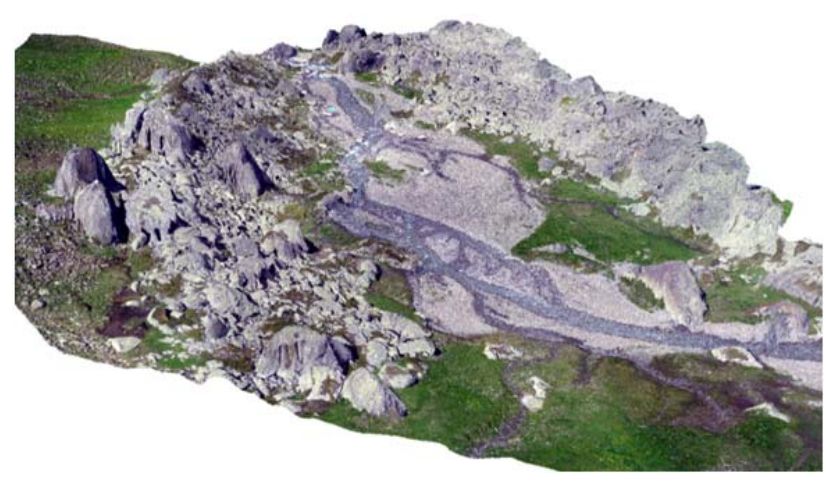

Figure 5: Resulting DSM Generated with TLS

\subsection{UAV Photogrammetry}

A Falcon 8 UAV (Ascending Technologies) with a Panasonic Lumix LX-3 digital compact camera was used to obtain aerial photographs of the site. The system allows one to upload the previously prepared flight plan to the on-board computer of the UAV. The flight planning allows setting the waypoints, the number of images to be taken at those waypoints and the viewing direction of the UAV and of the camera. This makes it possible to combine nadir and oblique images. Additionally, there is a possibility to setup a project suitable for selfcalibration where the UAV is rotating constantly by $90^{\circ}$ in order to obtain a good image network configuration.

A total of 15 ground control points were measured in the area. The points were marked as round targets on laminated A3 paper as seen in Figure 6, and measured with GNSS. The correction values for the GNSS positions were delivered using a static reference station. 


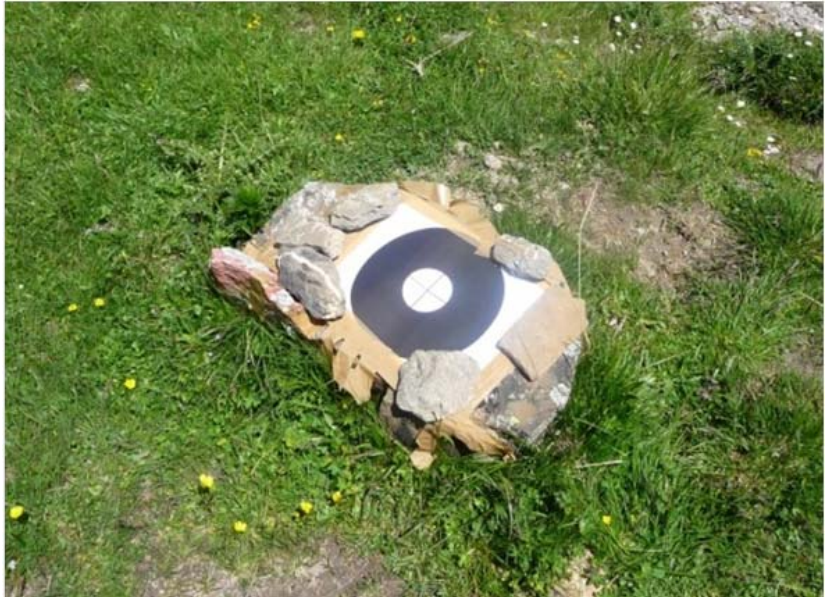

Figure 6: Ground Control Points for UAV Acquisition

Before the flight the camera was pre-calibrated via iWitness (Photometrix). The focusing is not done mechanically on the LX-3 but with a focus-by-wire system. Either due to vibrations or due to electronic interference the focus tends to slightly change over time, causing blurred images. Because there was no possibility to fix this problem onsite, it was decided to fly with the auto-focus enabled and then to do a self-calibration later on. While this could potentially impair precision and reliability, for this kind of project it was deemed to be accurate enough.

The project region was divided into a west and an east area. The east area was divided into four blocks - each block took about 40 minutes to fly and thus about three batteries were needed per block. The east area was done in one block and only one battery was needed to cover the block.

Over 190 images were taken of the area. The flight path of the UAV is shown in Figure 7. For each image the position and the orientation angles of the UAV was recorded using the on board GPS and IMU sensor. This information can be used as approximations for photogrammetric processing. The flying height of 60 metre equalled a pixel size of $2.5 \mathrm{~cm}$ in object space.

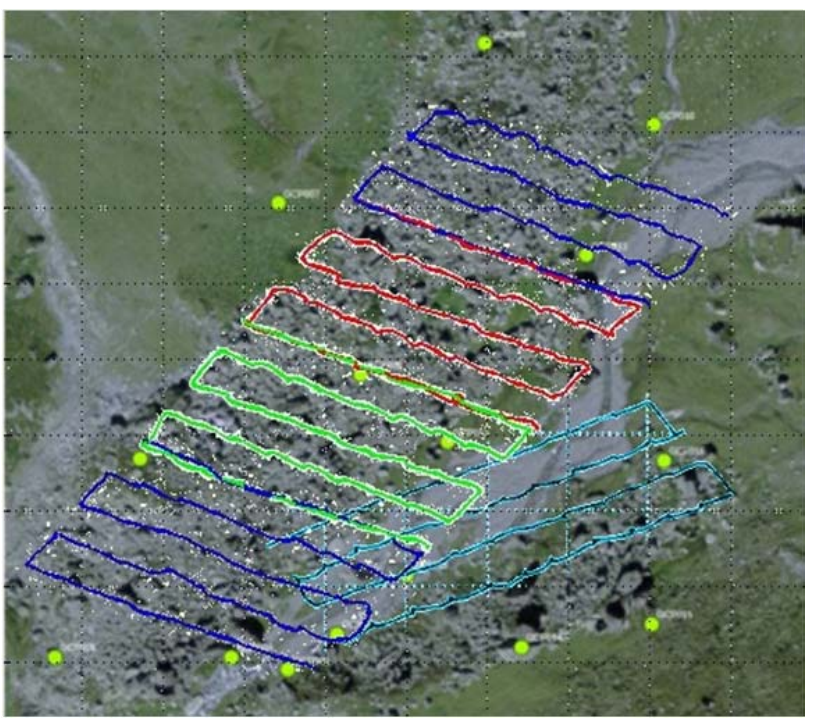

Figure 7: Flight Path of the UAV
The processing of the data was done using both Photomodeler (Eos Systems Inc.) and Leica Photogrammetry Suite (LPS, Leica Geosystems). Photomodeler allows for automated orientation of images without any approximations and without the need of ground control points. The model was initially georeferenced in Photomodeler using affine transformation. The orientation values were imported into LPS, where a combined bundle block adjustment was done. The adjustment yielded an RMS of 0.79 pixels. The ground control points had residuals of $2.5 \mathrm{~cm}$ in planimetry and $5.5 \mathrm{~cm}$ in height.

The result was a digital surface model with a resolution of $10 \mathrm{~cm}$ as well as an orthophoto (Figure 8).

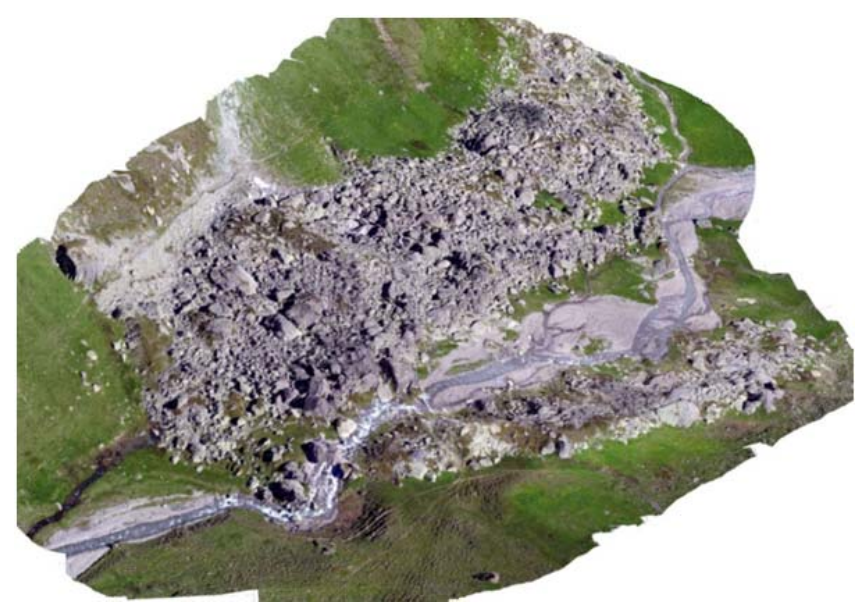

Figure 8: DSM Overlaid with its Orthophoto

\subsection{DSM Fusion}

The DSMs were co-registered through the ground control points that were measured in both models. The models were then fused by averaging them in the overlaying regions. In regions where only one system acquired data (i.e. the top of the boulders from the UAV), the data was kept as it was.

In Figure 9 (left) a section of the DSM generated by the laser scanner, draped with the orthophoto from the UAV is visualized. One can clearly see the irregularities on the bridge that come from a flat intersection angle between the laser beam and the bridge surface. Figure 9 (right) shows the DSM generated from the UAV images and draped with its orthophoto. The bridge is almost flat but the big stone on the left side of the bridge near the water shows problems because it is a slightly overhanging boulder. On the other hand, this boulder is better modelled in the TLS DSM.
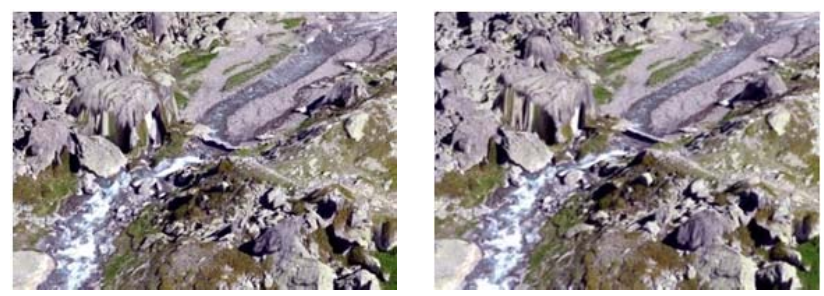

Figure 9: Comparison of a Section of the DTMs Left: DSM from TLS, Right: DSM from UAV

By combining the digital terrain models the strength of both measuring techniques can be exploited. As seen in Figure 10 in 
the combined DSM the bridge is flat and the boulder has correct dimensions.

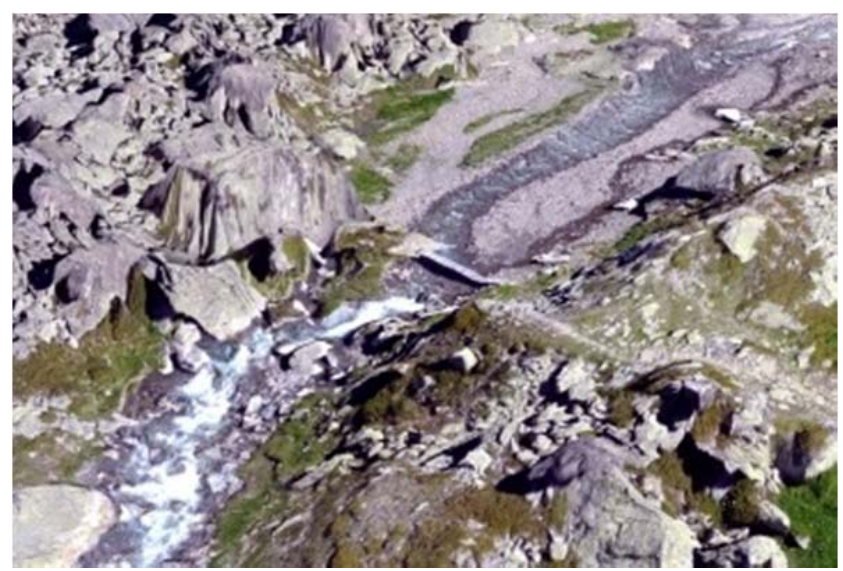

Figure 10: Section of the Combined DSM

Figure 11 shows differences between the TLS DSM and the UAV DSM.

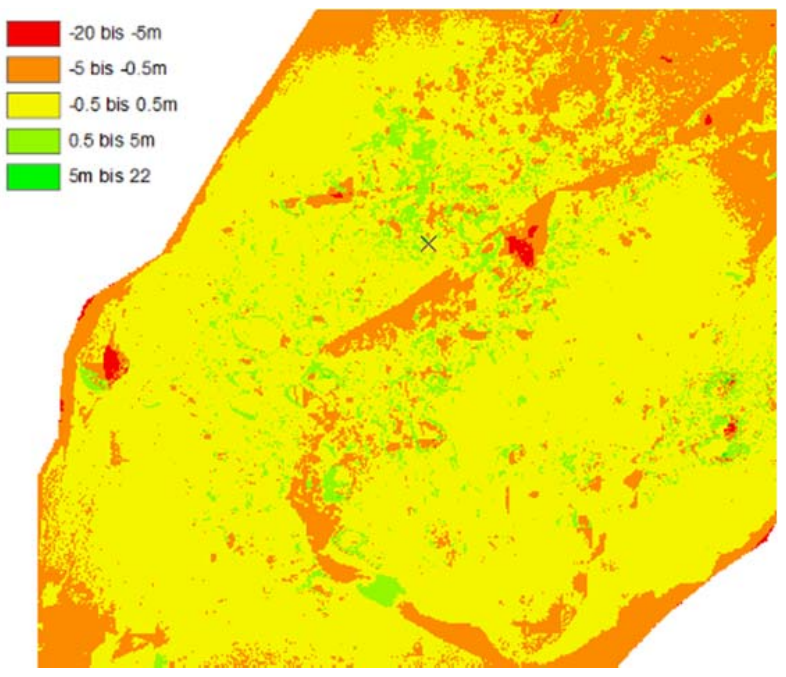

Figure 11: DSM Differences

In most parts the differences are smaller than 0.5 metres. The bigger differences come mostly from overhanging boulders where either the UAV or the laser scanner could not acquire any data and thus these regions were interpolated. In the middle of Figure 11 a seam is visible which denotes a larger difference. This difference comes from the mosaicking that was done to combine the UAV model from the east and the west part. To the right of this seam, there are other bigger differences, which are in the area of the small river. The water is difficult to match in the UAV images and also results in larger noise in the laser scans.

\section{MODELING OF STONE BOULDERS}

The goal of this step was to provide detailed models of 4 large boulders, which are of archaeological relevance. Under these boulders, excavations have been and will be carried out by the archaeologists from the University of Zurich. Thus the models can for example be used to document the current state, for visualisations or as a basis for finding new excavation spots.

\subsection{Terrestrial Laser Scanning}

To generate detailed boulder models, on the one hand a TLS Imager 5006i (Zoller + Fröhlich) was used. According to the manufacturer, the scan range is limited to 79 metre and must at least be $40 \mathrm{~cm}$. The noise level depends strongly on the reflectance of the scanned material. In our application, we expect maximal standard deviations of several millimetres. An industrial camera is fixed on top of the scanner and can be used to colour the scans.

To estimate the relative and absolute pose of each laser scan, white wooden spheres were used as tie and control points. At least 3 control points per boulder were measured using a referenced total station or differential GNSS. To get the relative pose of two scans using a Helmert transformation with 6 parameters ( 3 translation, 3 rotation) not less than 3 common tie points are to be used. An example of a scan configuration is demonstrated in Figure 12. On the right side of the image, the laptop to control the scanner and 2 tie point spheres can be seen.

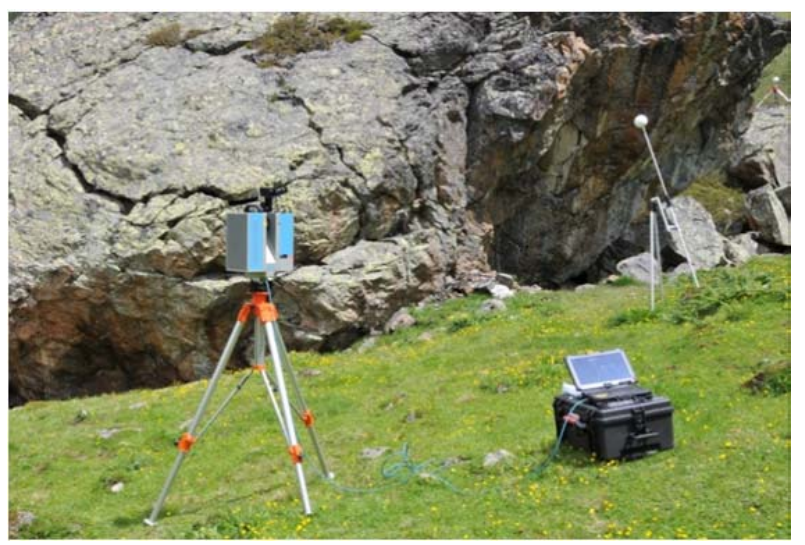

Figure 12: Scanning of the Boulders

The scans are primarily pre-processed in $\mathrm{Z}+\mathrm{F}$ Lasercontrol software (Zoller + Fröhlich). To transform the scans into the reference system, the scanned spheres have to be modelled and thus the centre of the sphere can be used as a tie point. This step can be accomplished semi-automatically. All tie points are used for the relative orientation of the scans. With the aid of the measurements from the total station and GNSS the absolute orientation can be performed. Based on the previously accomplished calibration of the camera, the colouring of the scans can be carried out automatically. Thus we get coloured point clouds containing 50 million points on average, which can be further processed, as described in Chapter 3.3.

\subsection{Close Range Photogrammetry}

Two professional Digital Single-lens Reflex (DSLR) cameras were used in order to obtain images of three stone boulders. The Nikon D3x is a full frame DSLR camera with a 24.5 Mega pixel CMOS sensor, whereas Nikon D2x has also a CMOS sensor, but of an APS-C format with a resolution of 12.4 Mega pixels. Together with both cameras, a set of three fixed-focus lenses was used: $18 \mathrm{~mm}, 24 \mathrm{~mm}$ and $35 \mathrm{~mm}$ focal length.

Before the image acquisition, each of the camera-lenses, focused at infinity, was calibrated using the Australis package (Photometrix), which consists of portable targets and calibration software. The calibration was performed in the field, which takes only 20-30 minutes, including taking the photographs of 
the portable targets (they are retro-reflective and require the use of a flash, see Figure 13), uploading images on the laptop and automatically performing the calibration. The uncertainty of the calibration did not exceed 0.18 pixels.

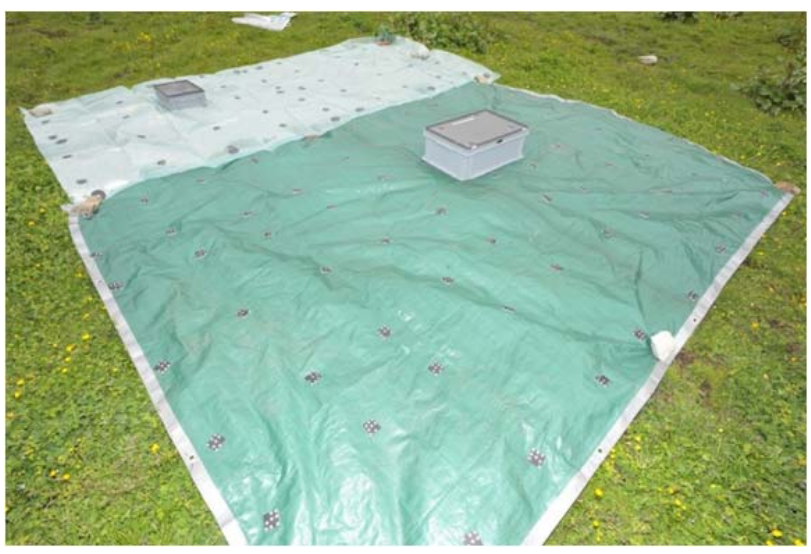

Figure 13: An In-Field Calibration Field with Australis RetroReflective Calibration Targets

A set of adhesive retro-reflective targets (Figure 14), attached to the boulders in approximately even spacing, was used for georeferencing. Their coordinates were determined with a total station, which has a special mode for measuring targets made of retro-reflective foil. Total stations were positioned on a fixed point, measured beforehand with GNSS, or using free-station mode.
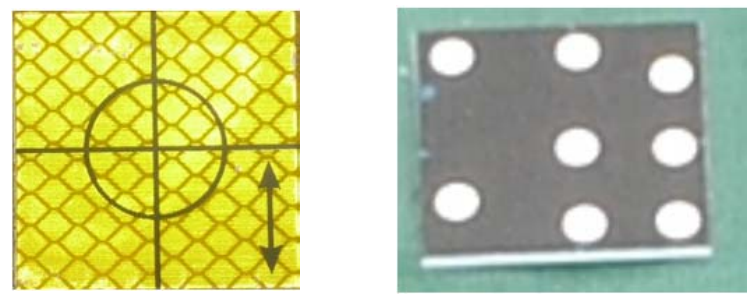

Figure 14: Retro-Reflective Targets

Left: for Georeferencing, Right: for Calibration

The images were acquired from such a distance that an accuracy of at least $2 \mathrm{~cm}$ could be achieved at the most distant object points, resulting in an average pixel size of approximately $3 \mathrm{~mm}$. What is more, the small distance to the object was also motivated by a need to fill the whole image frame with the object, in order to keep proper geometry of a photogrammetric network. Because of the poor illumination, the lower parts of the stone boulders had to be photographed with flash, which caused the retro-reflective targets to gleam, reducing the measurement accuracy.

The initial processing steps included sorting the large number of images, and small radiometric corrections. Further steps were carried out using Photomodeler Scanner software. The images were semi-automatically oriented: the coarse relative orientation was done manually. Then, having first exterior orientation parameters, it was possible to extract tie points automatically. The final RMSE of the bundle adjustment was 1.2 pixels.

After having the block of images oriented, it was possible to densely match image pairs and generate point clouds of the boulders, each having roughly 15 million points. The stone surface has good texture and Photomodeler dense matching performed reliably. The coloured point cloud was then further processed, as described in Chapter 3.3. An example point cloud, together with the camera stations (screen-shot from Photomodeler Scanner software) can be seen on Figure 15.

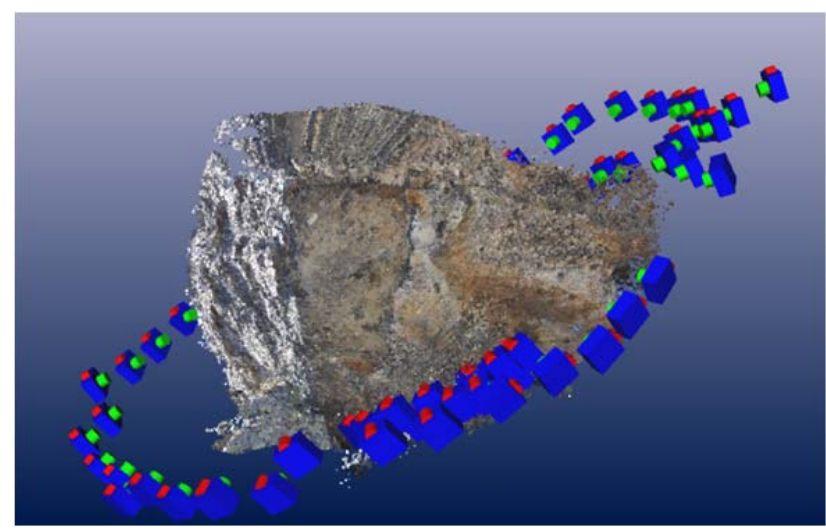

Figure 15: Example of the Point Cloud, Together with Camera Positions

\subsection{Point Cloud Processing}

In a first step, the noise of the point clouds from both methods (TLS and close-range photogrammetry) was reduced using Gaussian filtering in Geomagic software. Furthermore, irrelevant regions such as vegetation or neighbouring rocks were removed. Particularly the TLS point clouds include regions with exaggerated point density. To get consistent point spacing, these point clouds were thinned out to a resolution of $1 \mathrm{~cm}$.

Because of the special circumstances - demanding access, size and characteristics of the boulders - both point clouds exhibit numerous holes. For example, regions with low texture are better modelled by TLS, whereas bulges or cracks are hard to access and thus better suited for close-range photogrammetry. Therefore, there was a necessity to combine the two measurement techniques. Furthermore, due to the inaccessibility of the boulder tops, these parts were not covered with either method. The resulting gaps were filled using UAV-based photogrammetry.

The fusion of the three methods posed two main problems, as shown in Figure 16.

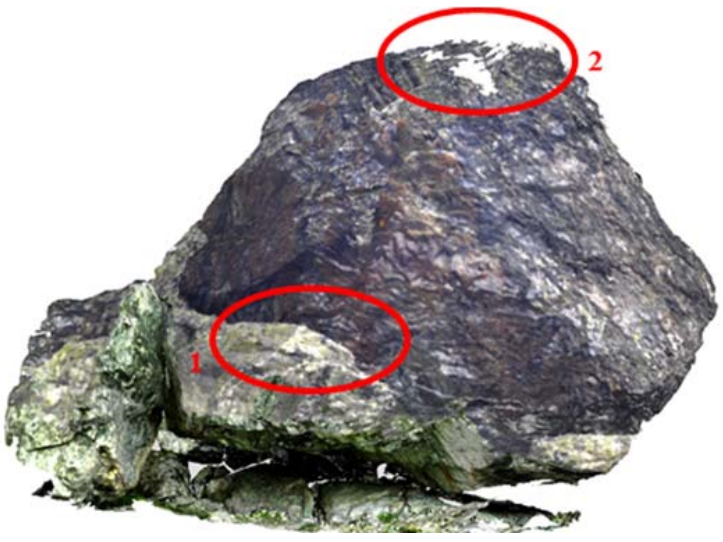

Figure 16: Fusion of Boulder Point Clouds 1: Radiometric Differences, 2: Holes on Boulder's Top 
First of all, the relative accuracy of all the methods was below the claimed value of $0.5 \mathrm{~cm}$. But due to the accuracy of the ground control points - based on GNSS measurements - the absolute accuracy was around $2 \mathrm{~cm}$. Therefore, the combination of the point clouds yields large deviations. Because there are large overlapping areas, this problem could be solved by applying an iterative closest point algorithm. The more problematic issue was the different radiometry of the three surveying methods. Ideally a radiometric adjustment should be done beforehand on the raw image data, but his was not done within the scope of this project.

After the successful fusion of the point clouds they were triangulated to a 3D mesh, which then was further improved by interpolating small holes and removing spikes. An example of the resulting boulder models combined with a raw mesh of the TLS DSM can be seen in Figure 17.

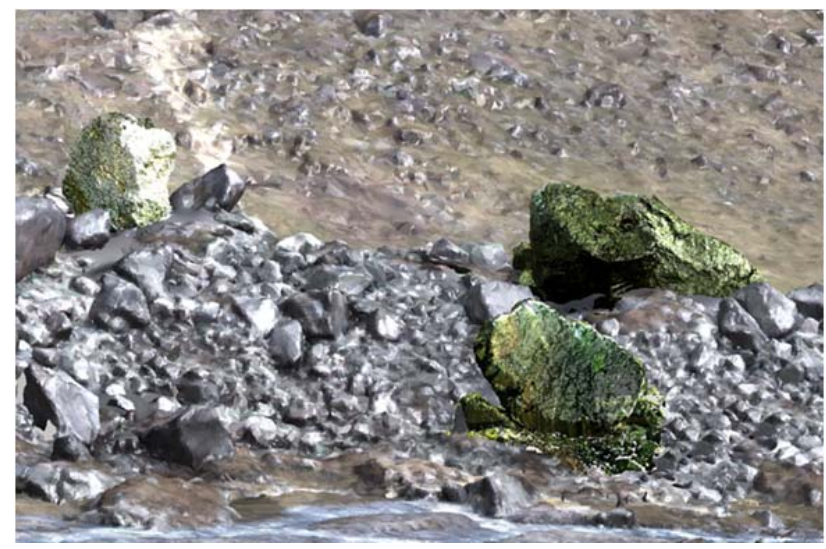

Figure 17: Modelled Boulders Combined with Raw Mesh from the TLS DSM

\section{CONCLUSIONS}

The aim of the present project was to obtain a digital surface model of the rock stream and models of 4 large boulders located in the Tasna Valley. The focus of this research has been on combining different data acquisition techniques. The digital surface model was obtained using a terrestrial laser scanning (TLS) and unmanned aerial vehicle (UAV) photogrammetry measurements. The stone boulders were reconstructed by combining the techniques of TLS, terrestrial and UAV photogrammetry. The various techniques and their combinations allowed evaluating them qualitatively and quantitatively.

For medium and large-sized areas, a common technique to acquire digital surface models is aerial photogrammetry or laser scanning. Using the UAV as an aerial platform proved its flexibility and ease of use. It was possible to cover the whole area $0.12 \mathrm{~km}^{2}$ with images in less than 3 hours. The additional workflow steps included measuring ground control points and post-processing (orienting images, model and orthophoto generation). The first step could be done (depending on the infrastructure available) in less than 1.5 hours, the latter depends not only on the software available, but also on the experience of the user. For an experienced user it might take less than a day, whereas for inexperienced one - even a week. As a result, a model of $2.5 \mathrm{~cm}$ planimetric accuracy and $5.5 \mathrm{~cm}$ height accuracy was obtained, which allowed generating an orthophoto with a resolution of $10 \mathrm{~cm}$. The radiometric quality of the orthophoto, which depends on the camera attached to the platform, was in this case very good.

Because of TLS range measurement limitations, to cover a large area with a sufficient point density, one has to use numerous scanner stations (in this study 30). Due to this requirement the data acquisition took 3 days and made this technique vulnerable to changing weather conditions, which can be a serious concern in such mountainous areas. A possible solution would be to use a long-range laser scanner with measurement ranges up to $2 \mathrm{~km}$. Notwithstanding, the point cloud resulting from TLS consists of 130 million points, with exaggerated point density in some regions. As a consequence of the poor radiometric quality delivered by the scanner system, the UAV images were preferred for orthophoto generation.

With the growing popularity of laser scanning technology in previous years, there has been a trend away from close-range photogrammetry for surveying applications. However, a continuing advantage of photogrammetric systems is their ability to work in almost all conditions, because the measuring system consists only of a camera and lens. Laser scanners are still bulky, need a solid mounting to the ground and yet, in order to acquire the data from a larger object, need many stations. This is again time consuming, while each time one changes the scanner position one has to orient it again. Moreover, in spite of vastly larger amount of points measured by laser-scanners in comparison to close-range photogrammetry, the radiometric quality of the texture still leaves a lot to be desired.

Nevertheless, during this study, laser-scanning outperformed close-range photogrammetry in terms of completeness. In order to acquire the data from all four stones, only few laser-scanner stations were needed, whereas close-range photogrammetry technique required taking large numbers of images, which significantly increased the processing time. Surprisingly, the lower parts of the boulders were acquired better with laserscanning, because of the lighting conditions (need to use a flash) and because of the low distance to object, which was not within the hyperfocal distance of the calibrated focal length. Finally, it proved advantageous to combine models acquired using both techniques, in order to achieve the best possible completeness and accuracy. As already mentioned in Chapter 3.3 the upper part of boulders was modelled by UAVphotogrammetry.

It has been shown that it is possible to achieve highly accurate and complete models of terrain and single objects using various common measuring techniques. As a general conclusion, we recommend combining different variants of photogrammetry and laser scanning, if possible, rather than restrict oneself to a single technique. The added flexibility is particularly valuable in remote environments, were challenges encountered during field work are less predictable.

\section{ACKNOWLEDGEMENTS}

All results were accomplished during a field course as part of the master program in Geomatics and Planning at ETH Zurich. The authors would like to thank all the students involved in this project. Special thanks go to the organizer of the field course David Grimm. 